\title{
THE GOTTLIEB GROUP OF FINITE LINEAR QUOTIENTS OF ODD DIMENSIONAL SPHERES
}

\author{
S. ALLEN BROUGHTON
}

(Communicated by Frederick R. Cohen)

\begin{abstract}
Let $G$ be a finite, freely acting group of homeomorphisms of the odd-dimensional sphere $S^{2 n-1}$. John Oprea has proven that the Gottlieb group of $S^{2 n-1} / G$ equals $Z(G)$, the centre of $G$. The purpose of this short paper is to give a considerably shorter, more geometric proof of Oprea's theorem in the important case where $G$ is a linear group.
\end{abstract}

In [G1], [G2] Gottlieb introduced subgroups $G_{n}(X) \subseteq \pi_{n}(X)$ of the homotopy groups of a connected space $X$, which have come to be known as the Gottlieb groups of $X$. The group $G_{1}(X)$ is usually referred to as the Gottlieb group of $X$ and has been extensively studied (cf., e.g., [Ga, L, P]). In [G1], Gottlieb provided the following characterization of $G_{1}(X)$. Let $\widetilde{X}$ be the universal cover of $X$ and identify $\Pi \simeq \pi_{1}(X)$ with the group of covering transformations in the usual way. Then, we have

Proposition 1. The Gottlieb group, $G_{1}(X)$, is the subgroup of $\Pi$ consisting of all $z$ which are equivariantly homotopic to the identity.

Remark. To show that $z$ is in $G_{1}(X)$ we must find a homotopy $L_{t}: \tilde{X} \rightarrow \tilde{X}$, $0 \leq t \leq 1$, such that

$$
L_{0}=i d, \quad L_{1}=z
$$

and

$$
L_{t} g=g L_{t}, \quad \text { for all } g \in \Pi \text { and for } 0 \leq t \leq 1 \text {. }
$$

From this characterization it is clear that the Gottlieb group is a characteristic subgroup, lying in the centre of $\Pi$. In this paper we prove the following theorem.

Theorem. Let the finite group $G$ act freely and linearly on the odd-dimensional sphere $S^{2 n-1}$. Then, the Gottlieb group of the quotient space $S^{2 n-1} / G$ is isomorphic to $Z(G)$, the centre of $G$, under the canonical isomorphism of $G$ with $\pi_{1}\left(S^{2 n-1} / G\right)$.

Received by the editors January 8, 1990 .

1980 Mathematics Subject Classification (1985 Revision). Primary 55Q52, 57S17, 57S25.

Key words and phrases. Gottlieb group, linear group actions. 
In [O], J. Oprea has proven a more general theorem in which it is only assumed that $G$ is a finite, freely acting group of homeomorphisms of $S^{2 n-1}$. The methods in [O] use rather complicated algebro-topological arguments because of the general nature of the action. The sole purpose of this paper is to show that if we restrict our attention to linear actions, a much simpler, geometric proof is possible. Before proceeding to the proof, we recall the following fact about linear representations of a finite group on a real vector space. For all the results on linear representations that we use, we refer to Isaacs's monograph [I1].

Proposition 2. Let the finite group $G$ act linearly and irreducibly, over $\mathbf{R}$, on the real vector space $V$. Let $\operatorname{Cent}_{G}(V)$ denote the commuting algebra of the $G$-action on $V$, i.e., the algebra of endomorphisms of $V$ which commute with $G$. Then, $\operatorname{Cent}_{G}(V)$ is a division algebra isomorphic to one of $\mathbf{R}, \mathbf{C}$, or $\mathbf{H}$ (quaternions). Furthermore, $\operatorname{Cent}_{G}(V) \simeq \mathbf{R}$ if and only if the representation of $G$ on $V$ is absolutely irreducible, i.e., the complex representation of $G$ on $\mathbf{C} \otimes V$ is irreducible.

Proof of theorem. Suppose that the free action of $G$ on the odd-dimensional sphere $S^{2 n-1}$ is induced by an orthogonal linear representation on the $2 n$ dimensional real vector space $V$. In the notation of Proposition 1, we take $X=S^{2 n-1} / G, \tilde{X}=S^{2 n-1}$, and $\Pi=G$. To prove the theorem we must produce, for each $z \in Z(G)$, a homotopy $L_{t}$ satisfying (1) and (2) above. If $G$ consists of the identity map and the antipodal map, then a homotopy from the identity map to the antipodal map may be constructed by letting $L_{t}$ be a path from $I$ to $-I$ in the unitary matrix group $U(n)$, which is path-connected. We may now assume that $G$ is not this group.

Let $g \in G$ have order $n$, the eigenvalues of $g$ are all primitive $n$th roots of unity, otherwise some nonidentity power of $g$ fixes a point. From this condition on the eigenvalues we get

(3) The only possible involution in $G$ is the antipodal map: $v \rightarrow-v$.

From the freeness of the $G$-action we also get

(4) If $W \subseteq V$ is a nonzero $G$-invariant subspace, then the restricted representation of $G$ on $W$ is faithful.

Write $V=V_{1} \oplus \cdots \oplus V_{s}$, an orthogonal direct sum of irreducible $G$-invariant subspaces over $\mathbf{R}$. Suppose that the commuting algebra of one or more of the $V_{i}$ 's is $\mathbf{R}$. Then, by (3), (4), and Proposition 2, $G$ is a group with only one involution and an absolutely irreducible faithful real representation. First, J. Malzan [M] and then M. Isaacs [I2] (using simpler methods) have shown that such a group has order 2 . In this case, $G$ would consist of the identity map and the antipodal map, but we have already handled this possibility.

Let $z \in Z(G)$. For $1 \leq i \leq s$, let $L_{t}^{i}$ be a path in $\operatorname{Cent}_{G}\left(V_{i}\right)$ starting at the identity and ending at $\left.z\right|_{V_{i}}$. Since we may assume that all of the centralizers $\operatorname{Cent}_{G}\left(V_{i}\right)$ are division algebras of real dimension at least two, we may 
construct the paths so that for each $t$ and $i$ the map $L_{t}^{i}$ is an invertible linear transformation of $V_{i}$. Define the homotopy $L_{t}$ by

$$
L_{t}(v)=M_{t}(v) /\left|M_{t}(v)\right|, \quad \text { for } v \in S^{2 n-1},
$$

where

$$
M_{t}(v)=L_{t}^{1}\left(v_{1}\right)+\cdots+L_{t}^{s}\left(v_{s}\right)
$$

II denotes the norm in $V$, and $v=v_{1}+\cdots+v_{s}$ is the decomposition of $v \in V$ induced by the decomposition of $V$ into $G$-invariant subspaces. By construction, each $M_{t}$ is invertible, so $L_{t}$ is well-defined. Since the $V_{i}$ 's are $G$-invariant, then the equivariance condition (2) holds with $L_{t}$ replaced by $M_{t}$. Since the norm is $G$-invariant and for each $g \in G, g M_{t}(v)=M_{t}(g v)$, then $\left|M_{t}(v)\right|=\left|g M_{t}(v)\right|=\left|M_{t}(g v)\right|$. It now follows that (1) and (2) hold for all $L_{t}$. All is now proven.

\section{REFERENCES}

[G1] D. Gottlieb, A certain subgroup of the fundamental group, Amer. J. Math. 87 (1965), 840856.

[G2] _ Evaluation subgroups of homotopy groups, Amer. J. Math. 91 (1969), 729-755.

[Ga] T. Ganea, Cyclic homotopies, Illinois J. Math. 12 (1968), 1-4.

[I1] M. Isaacs, Characters of finite groups, Academic Press, New York, 1976.

[I2] $\quad$, Real representations of groups with a single involution, Pacific J. Math. 71 (1977), 463-464.

[L] G. Lang, Evaluation subgroups of factor spaces, Pacific J. Math. 42 (1972), 701-709.

[M] J. Malzan, On groups with a single involution, Pacific J. Math. 57 (1975), 481-489.

[O] J. Oprea, Finite group actions on spheres and the Gottlieb group, Cleveland State University, preprint.

[P] J. Pak, On the fibered Jiang spaces, Contemp. Math. 72 (1988), 179-181.

Department of Mathematics, Cleveland State University, Cleveland, Ohio 44115 